\title{
Novos registros de Omalonyx matheroni (Pontiez \& Michaud, 1835) (Mollusca, Gastropoda, Succineidae) para os Estados de São Paulo e Paraná, Brasil
}

\author{
Janine Oliveira Arruda ${ }^{1 *}$ \\ Daniel Pereira ${ }^{2}$ \\ Paulo Eduardo Aydos Bergonci ${ }^{2}$ \\ Cíntia Pinheiro dos Santos ${ }^{2}$ \\ Maria Cristina Dreher Mansur ${ }^{2}$ \\ 'Laboratório de Malacologia, Museu de Ciências e Tecnologia \\ Pontifícia Universidade Católica do Rio Grande do Sul \\ Avenida Ipiranga 6681, prédio 40, CEP 90619-900, Porto Alegre - RS, Brasil \\ ${ }^{2}$ Centro de Ecologia, Universidade Federal do Rio Grande do Sul \\ Av. Bento Gonçalves, 9500, Setor 4, Prédio 43422, Sala 102, \\ CEP 91540- 000, Porto Alegre - RS, Brasil \\ *Autor para correspondência \\ arrudajo@gmail.com
}

Submetido em 08/11/2008

Aceito para publicação em 17/12/2008

\section{Resumo}

Omalonyx matheroni é um gastrópode sucineídeo que ocorre nas macrófitas aquáticas e vegetações adjacentes de planície de inundação de rios, em lagos e açudes. Registra-se a ocorrência dessa espécie para os municípios de Ibitinga (SP) e Paranaguá (PR), estendendo sua distribuição mais ao sul da América do Sul. Até o presente momento, a espécie tinha sido registrada para Demerara (Guiana), Zanderij e Belwaarde (Suriname), Guiana Francesa, Peru, Limoncocha (Equador), Amazonas, Pará, Pernambuco, Rio de Janeiro e Minas Gerais (Brasil), além das ilhas de Guadalupe e Trinidade.

Unitermos: Omalonyx matheroni, Ibitinga, Paranaguá, macrófitas aquáticas

\section{Abstract}

New records of Omalonyx matheroni (Pontiez \& Michaud, 1835) (Mollusca, Gastropoda, Succineidae) for the São Paulo and Paraná States. Omalonyx matheroni is a succineid gastropod that lives on aquatic macrophytes and on emergent vegetation in the wetlands of inner deltas, lakes and dikes. Occurrences of this species were recorded in the municipalities of Ibitinga (SP) and Paranaguá (PR), broadening its distribution southwards in South America. Until now this species had been recorded in Demerara (Guiana), Zanderij and Belwaarde (Suriname), Guiana Francesa, Peru, Limoncocha (Equador), Amazonas, Pará, Pernambuco, Rio de Janeiro and Minas Gerais (Brazil), as well as on the islands of Guadalupe and Trinidade.

Key words: Omalonyx matheroni, Ibitinga, Paranaguá, aquatic macrophytes 
Omalonyx d'Orbigny, 1837 é representado por lesmas, de aproximadamente dois centímetros de comprimento, que possuem uma concha externa reduzida, achatada e unguiforme, e um padrão de coloração com duas linhas longitudinais negras e manchas negras sobre todo o corpo, incluindo o manto (Arruda et al., 2006) (Figura 1). Apresentam um processo de limacização bastante desenvolvido, quando comparados aos demais gêneros de Succineidae (Tillier, 1984). Exibem hábito anfíbio, vivendo associados à macrófitas aquáticas flutuantes (Eichhornia crassipes, Salvinia auriculata e Pistia stratiotis) e emergentes (Paspalum sp. e Eryngium sp.) em banhados, planícies inundáveis de rios, açudes, arroios e lagos (Pereira et al., 2000a e b; Barker, 2001; Arruda, 2007). Uma ocorrência atípica foi registrada por Garcia et al. (2006), em um campo de cultivo de capim gordura (Pennisetum purpureum) em Manaus (AM - Brasil). As espécies de Omalonyx apresentam distribuição neotropical, com ocorrência para as ilhas do Caribe, ilha de Juan Fernandez, América Central e do Sul (Patterson, 1971). Não constituem um grupo com vasta representabilidade em coleções científicas, conforme comentado por Hylton Scott e Lapuente (1968). Segundo esses autores, por serem relativamente pequenos, desprovidos de atrativos e por encontraremse entre a vegetação aquática, são encontrados apenas quando expressamente procurados.
Omalonyx matheroni (Potiez \& Michaud, 1835), segundo Tillier (1981), ocorre em algumas ilhas nas Pequenas Antilhas e, na América do Sul, de Caracas (Venezuela) ao Rio de Janeiro (Brasil). Externamente é bastante semelhante às demais espécies do gênero. A concha figura poucas informações sistemáticas. Apenas por meio do estudo detalhado da anatomia interna do sistema reprodutório é possível determinar a espécie com precisão. Arruda et al. (2006) representaram o sistema reprodutório e listaram como caracteres diagnósticos a presença de ovariotestis hemisférico, superfície externa do epifalo lisa, superfície interna do evertofalo com papilosidades elípticas e oviduto livre três vezes o comprimento do oviduto palial. Esta espécie é registrada pela primeira vez para os Estados de São Paulo e Paraná (Brasil). Os seguintes lotes foram examinados: SAO PAULO, Ibitinga, usina hidrelétrica de Ibitinga, margem do rio Tietê, 13/V/2008, 1 spec., Pereira, Bergonci e Santos leg. (MCP 9120); PARANA, Paranaguá, rio Inferninho, 17/V/1967, 1 spec., Biasi e Jay leg. (MZUSP 18440) (Figura 2). Estes registros ampliam a área de distribuição de O. matheroni mais ao sul na América do Sul. Provavelmente esta espécie faz limite com Omalonyx convexus (Heynemann, 1868), revalidada por Arruda e Thomé (2008), e cuja ocorrência mais ao norte está registrada para o Estado de Santa Catarina (Agudo, 2004).

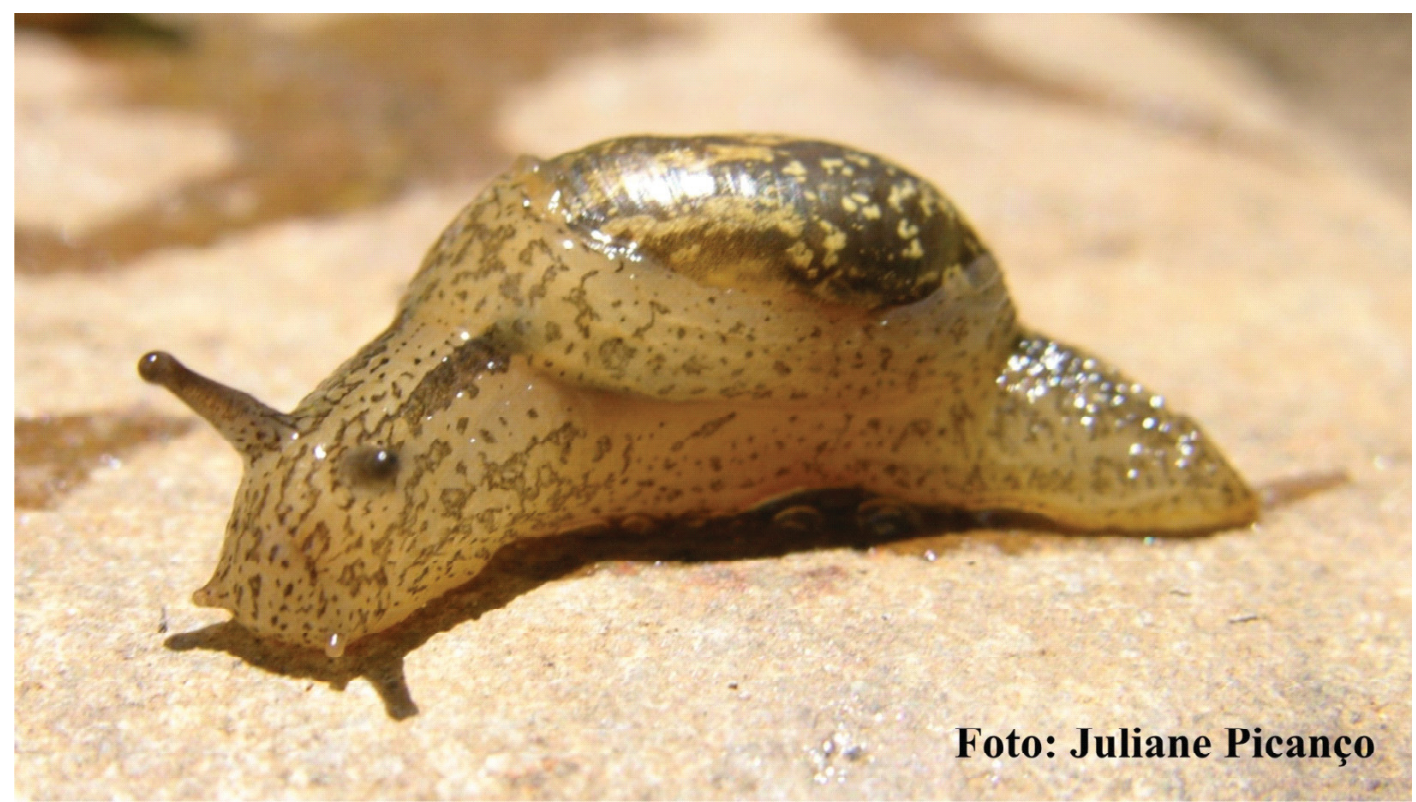

Figura 1: Exemplar de Omalonyx d'Orbigny, 1837. 


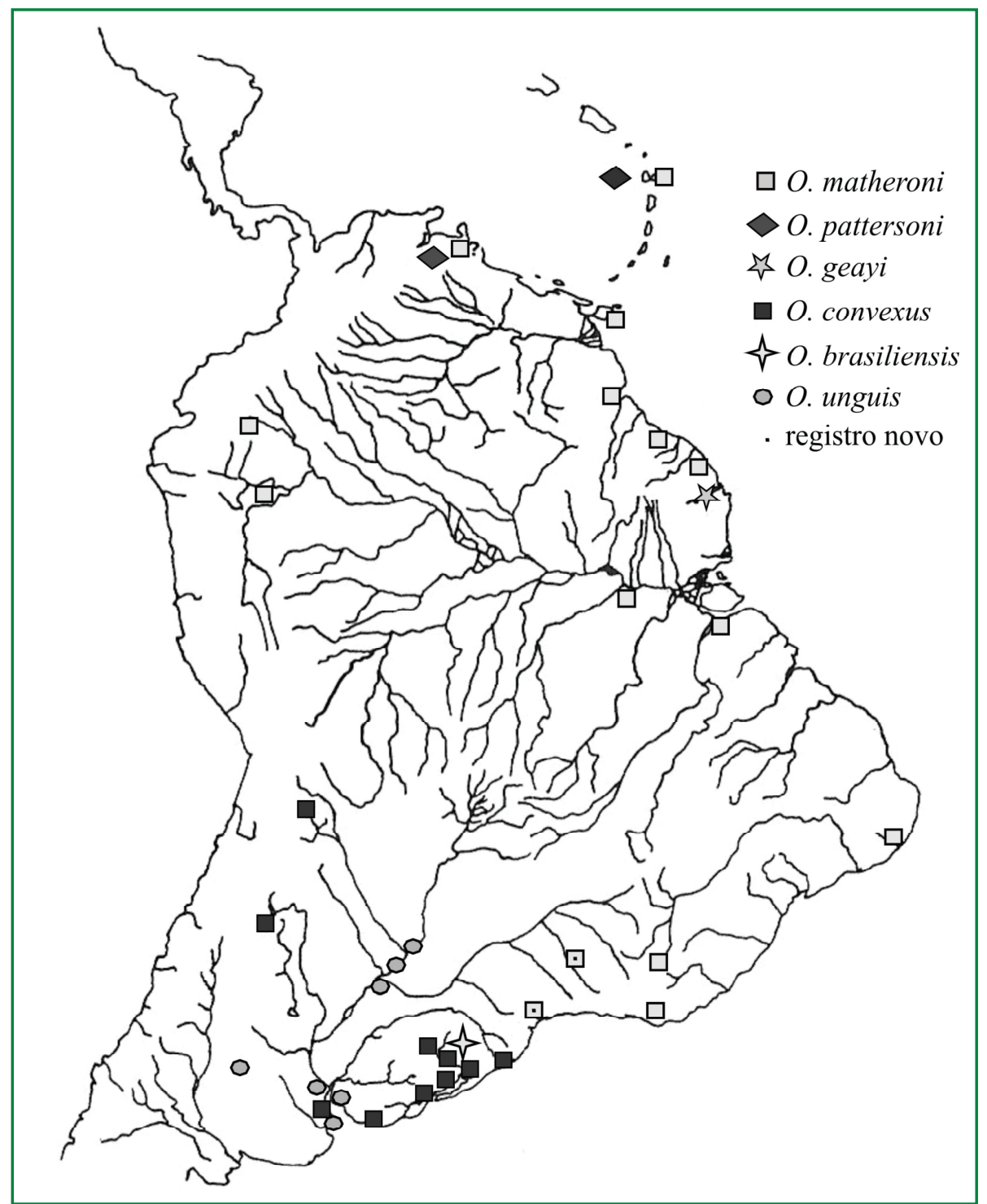

Figura 2: Distribuição das espécies de Omalonyx d'Orbigny, 1837, adaptado de Tillier (1981).

\section{Abreviaturas}

MCP: Museu de Ciências da Pontifícia Universidade Católica do Rio Grande do Sul (Porto Alegre, RS) MZUSP: Museu de Zoologia da Universidade de São Paulo (São Paulo, SP)

\section{Agradecimentos}

Ao Dr. Luiz R. L. Simone pelo empréstimo do material do MZUSP; aos consultores anônimos pela leitura cuidadosa e excelente crítica.

\section{Referências}

Agudo, I. 2004. Preliminary report on the freshwater mollusk fauna of Mampituba river basin, Santa Catarina State, Southern Brazil. Ellipsaria, 6 (1): 10.
Arruda, J. O. 2007. Sistemática e ecologia de espécies de Omalonyx (Mollusca, Gastropoda, Succineidae) no estado do Rio Grande do Sul. Dissertação de Mestrado, Pontifícia Universidade Católica do Rio Grande do Sul, Brasil, 57pp.

Arruda, J. O.; Gomes, S. R.; Ramirez, R.; Thomé, J. W. 2006. Morfoanatomia de duas espécies do gênero Omalonyx (Mollusca, Gastropoda, Succineidae) com novo registro para Minas Gerais, Brasil. Biociências, 14 (1): 61-70.

Arruda, J. O.; Thomé, J. W. 2008. Revalidation of Omalonyx convexus and emendation of the type locality of Omalonyx unguis (Mollusca, Gastropoda, Succineidae). Archiv für Molluskenkunde, 137 (2): 159-166. 
Barker, M. G. 2001. Gastropods on land: Phylogeny, diversity and adaptive morphology. In: Barker, M. G. (Ed.). The biology of terrestrial mollusks. CABI Publishing, New York, USA, p.1-146.

Garcia, M.; Pimpão, D. M.; Vidigal, T. H. D. A.; Brito, M.; Coscarelli, D.; Silva, F. 2006. Ocorrência de Omalonyx sp. (Gastropoda: Pulmonata: Succineidae) como praga agrícola na região de Manaus. Resumos do I Simpósio da SBPC no Amazonas, Manaus, Brasil, p.48.

Hylton Scott, M. I.; Lapuente, E. 1968. Valor diagnostico de la radula para especies del genero Homalonyx Orbigny (Gastropoda Succineidae). Neotropica, 14 (44): 49-56.

Pereira, D.; Konrad, H. G.; Paloski, N. I. 2000a. Gastrópodos límnicos da bacia do rio Camaquã, RS, Brasil. Acta Biologica Leopoldensia, 22 (1): 55-66.
Pereira, D.; Veitenheimer-Mendes, I. L.; Mansur, M. C. D.; Silva, M. C. P. 2000b. Malacofauna límnica do sistema de irrigação do arroio Capivara, Triunfo, RS, Brasil. Biociências, 8 (1): 137-157.

Patterson, C. M. 1971. Taxonomic studies of the land snail family Succineidae. Malacological Review, 4: 131-202.

Tillier, S. 1981. South American and Juan Fernández succineid slugs (Pulmonata). Journal of Molluscan Studies, 47 (2): 125-146.

Tillier, S. 1984. Patterns of digestive tract morphology in the limacisation of helicarionid, succineid and athoracophorid snails and slugs (Mollusca: Pulmonata). Malacologia, 25 (1): 173-192. 